\title{
Adaptação transcultural para o português brasileiro da escala effort-reward imbalance: um estudo com trabalhadores de banco ${ }^{1}$
}

\author{
Luiz Sérgio Silva² e Sandhi Maria Barreto²
}

Como citar Silva LS, Barreto SM. Adaptação transcultural para o português brasileiro da escala effort-reward imbalance: um estudo com trabalhadores de banco. Rev Panam Salud Publica. 2010;27(1):32-6.

Palavras-chave

Objetivo. Descrever a adaptação transcultural da escala effort-reward imbalance para o português brasileiro e analisar a validade e a confiabilidade da adaptação.

Método. Foi utilizado o método da tradução/retrotradução. A consistência interna, a confiabilidade teste-reteste e a estrutura fatorial da escala adaptada foram testadas em uma amostra de 100 trabalhadores de um banco estatal brasileiro, de ambos os sexos, com diferentes idades e níveis educacionais.

Resultados. A versão adaptada foi denominada escala de desequilíbrio esforço-recompensa. Dos 100 trabalhadores, $62 \%$ eram do sexo masculino. A idade variou entre 23 e 65 anos (média de 39 anos); a escolaridade, entre 3 e 27 anos (média de 15 anos); e o tempo de trabalho na empresa, entre 1 e 31 anos (média de 11 anos). O alfa de Cronbach para as três dimensões da escala - esforço, recompensa e comprometimento excessivo - foi de 0,70,0,95 e 0,86, respectivamente. Os coeficientes de correlação intraclasse para as mesmas dimensões foram 0,82, 0,96 e 0,91. A análise fatorial manteve todos os itens iniciais da escala e foi consistente com os componentes de construto do modelo teórico.

Conclusão. Os resultados indicam que a adaptação transcultural para português brasileiro da escala de desequilibrio esforço-recompensa foi bem-sucedida e é adequada para avaliar esse desequilibrio em ambientes de trabalho.

Estresse psicológico; carga de trabalho; recompensa; satisfação no trabalho; testes psicológicos; Brasil.

Sabe-se atualmente que as condições de trabalho em que há significativo desequilíbrio entre o esforço despendido e a recompensa recebida são extremamente estressantes e podem acarretar proble-

O autor da escala, Johannes Siegrist, autorizou a adaptação transcultural em 22 de janeiro de 2007.

2 Universidade Federal de Minas Gerais, Faculdade de Medicina, Medicina Preventiva. Programa de Pós-Graduação em Saúde do Adulto. Grupo de pesquisa em doenças crônicas e ocupacionais (Germinal). Enviar correspondência a Luiz Sérgio Silva no seguinte endereço: Avenida Professor Alfredo Balena 190, Santa Efigênia, CEP 30130-100, Belo Horizonte, MG, Brasil. E-mail: luizsergios@yahoo. com.br mas de saúde, particularmente em pessoas com maior dificuldade de adaptação (1). Já foram relatadas associações entre situações estressantes de desequilíbrio, esforço e recompensa no trabalho e aumento do risco de doenças cardiovasculares, distúrbios psiquiátricos e alcoolismo em diversos grupos ocupacionais (2-6). Levando esses aspectos em consideração, a pesquisa sobre o desequilíbrio entre esforço e recompensa - mais especificamente o modelo de desequilíbrio esforço-recompensa (effort-reward imbalance model, ERI) - e sobre a sua relação com a saúde procura entender a contri- buição dos fatores sociais e psicológicos para a saúde e a doença humana.

O modelo ERI baseia-se na reciprocidade de intercâmbio na vida profissional. De acordo com o modelo, uma pessoa com maior necessidade de controle responde de maneira inflexível às situações de trabalho que exigem muito esforço e oferecem baixa recompensa, acarretando estresse e predispondo ao adoecimento $(2,7)$. Hasselhorn et al. (8) afirmam que situações de estresse crônico no trabalho podem predispor o trabalhador a iniciar distúrbios autonômicos e respostas neuroendócrinas desfavoráveis. O modelo 
teórico da escala de desequilíbrio entre esforço e recompensa é usado para medir o nível de estresse a que esse trabalhador está exposto.

Dois componentes de esforço e recompensa são contemplados no modelo: um extrínseco e um intrínseco. O componente extrínseco tem como foco as condições do trabalho indicativas do esforço (demandas, obrigações) e as recompensas (dinheiro, apoio, oportunidades na carreira e segurança). O componente intrínseco enfoca o estilo pessoal de ajustamento, denominado comprometimento excessivo (overcommitment). Esse comprometimento excessivo define um conjunto de atitudes, comportamentos e emoções que refletem esforço excessivo combinado com forte desejo de ser aprovado e estimado. Pessoas com esse estilo pessoal tendem a exagerar seus esforços (8).

A epidemiologia social se propõe a desenvolver questionários e escalas psicométricas que possam ser utilizadas para pesquisas em larga escala (1). O modelo ERI é construído a partir de um questionário autorrespondido, contendo 23 itens em sua versão reduzida, com respostas que utilizam escala do tipo likert. Entretanto, o seu uso em contextos internacionais é limitado pela língua, exigindo, portanto, uma adaptação transcultural.

Para que um instrumento seja adaptado adequadamente para outro idioma, Heredman et al. (9) ressaltam a necessidade de se levar em conta pelo menos algumas dimensões de equivalência: a equivalência conceitual, a equivalência semântica e a equivalência operacional, que significa a preservação do formato, das instruções, da forma de aplicação e dos métodos de medida. Se as duas escalas forem equivalentes de acordo com essas três dimensões, considera-se que existe equivalência funcional entre as duas versões e que ambas medem os mesmos conceitos em culturas distintas.

O presente trabalho tem como objetivo descrever a adaptação para o português brasileiro da versão resumida da escala effort-reward imbalance, originalmente aplicada em inglês e adaptada para diversos idiomas, além de testar a validade de construto e a confiabilidade dessa adaptação.

\section{MATERIAIS E MÉTODOS}

A adaptação da escala effort-reward imbalance, denominada em português brasileiro de escala desequilíbrio esforçorecompensa, foi feita a partir da versão original em inglês pelos autores deste artigo. Duas traduções independentes foram reunidas em uma versão de consenso que foi aplicada a um grupo de 20 pessoas que expressaram suas dificuldades de entendimento e fizeram sugestões para melhorias. Essa versão de consenso foi ajustada conforme as sugestões e novamente avaliada pelos dois pesquisadores, sendo aplicada a um grupo de 30 bancários voluntários, de ambos os sexos e com escolaridade diversa. Nessa segunda avaliação, foram aferidos o tempo gasto para responder todas as perguntas e possíveis dificuldades de compreensão.

Uma nova versão foi feita e apresentada a um tradutor independente, nativo de país de língua inglesa (Estados Unidos), que fez a retrotradução para o inglês americano e comparou o produto da retrotradução ao original para ajustes finais. A versão final do questionário foi então aplicada em estudo do tipo testereteste a uma população de 100 trabalhadores de um banco, de ambos os sexos, com escolaridade e inserção ocupacional diversas.

O questionário final contém 23 questões, organizadas em três escalas unidimensionais: esforço (seis itens), recompensa (11 itens) e comprometimento excessivo (seis itens). A escala de recompensa pode ser subdividida em três subescalas: reconhecimento, segurança no trabalho e promoção no emprego/salário, composta pelos itens 11, 14, 16 e 17. Nas escalas de esforço e recompensa as respostas variam no grau de concordância ou discordância, com escores que vão de 1 a 5 . Na escala de comprometimento excessivo, as respostas variam entre discordar fortemente e concordar fortemente, com escores entre 1 e 4 .

Para análise da consistência interna foi usado o alfa de Cronbach, que mede a homogeneidade dos itens da escala, ou seja, se esses itens medem os mesmos atributos. Para verificar a confiabilidade teste-reteste, foi usado o coeficiente de correlação intraclasse, que reflete a correlação e a concordância entre as duas aplicações do mesmo instrumento. A estrutura do questionário foi avaliada por análise fatorial (rotação varimax) com extração dos fatores principais, permanecendo no modelo aqueles com valor próprio (eigenvalue) superior a 1. A análise fatorial separa as variáveis em grupos correlacionados, que medem a mesma dimensão; essa medida identifica aquelas questões que são linearmente correlacionadas.

O projeto foi aprovado pelo Comitê de Ética em Pesquisa da Universidade Federal de Minas Gerais, Brasil, tendo sido obtido o consentimento livre e esclarecido de todos os participantes.

\section{RESULTADOS}

Em relação ao grupo pesquisado, $62 \%$ dos participantes eram do sexo masculino. A idade variou entre 23 e 65 anos, com média de 39 anos. A escolaridade variou entre 3 e 27 anos de estudo, tendo como média 15 anos. Todos os entrevistados estavam empregados no momento da pesquisa, com tempo de trabalho na empresa variando de 1 a 31 anos, com média de 11 anos. Dos pesquisados, $64 \%$ eram concursados e $7 \%$ trabalhavam em turno e exerciam outra atividade remunerada além do emprego declarado. A média de horas semanais trabalhadas foi de 43,6 horas.

A tabela 1 apresenta os resultados da análise da confiabilidade teste-reteste e da consistência interna da escala. Os co-

TABELA 1. Escores médios, alfa de Cronbach e coeficientes de correlação intraclasse para as dimensões, validação da escala desequilíbrio esforço-recompensa, Brasil, 2007 a 2008

\begin{tabular}{lccc}
\hline \multicolumn{1}{c}{ Dimensão } & Escore médio (DP) & Alfa & $\begin{array}{c}\text { Coeficiente de correlação } \\
\text { intraclasse (IC95\%) }\end{array}$ \\
\hline Esforço & $12,43(4,29)$ & 0,70 & $0,82(0,75$ a 0,87$)$ \\
Recompensa & $39,22(14,86)$ & 0,95 & $0,96(0,95$ a 0,97$)$ \\
Comprometimento excessivo & $13,64(4,01)$ & 0,86 & $0,91(0,89$ a 0,94$)$ \\
\hline
\end{tabular}


TABELA 2. Matriz fatorial varimax da adaptação para o português brasileiro da escala desequilíbrio esforço-recompensa, Brasil, 2007 a 2008

\begin{tabular}{|c|c|c|c|c|c|}
\hline \multirow[b]{2}{*}{ Escala e item ${ }^{b}$} & \multicolumn{5}{|c|}{ Fatora } \\
\hline & 1 & 2 & 3 & 4 & 5 \\
\hline \multicolumn{6}{|l|}{ Esforço } \\
\hline 1. Eu me sinto constantemente pressionado para fazer muito trabalho em pouco tempo & & 0,337 & & 0,738 & \\
\hline 2. Constantemente interrompem e atrapalham meu trabalho & & 0,344 & & 0,546 & \\
\hline 3. Eu tenho muitas responsabilidades no meu trabalho & & 0,526 & & 0,480 & \\
\hline 4. Eu sou pressionado com frequência a trabalhar além do horário & & & & 0,677 & \\
\hline 5. Meu trabalho exige esforço físico & & & & & 0,801 \\
\hline 6. Nos últimos anos, meu trabalho tem se tornado cada vez mais exigente & & 0,576 & & 0,319 & \\
\hline \multicolumn{6}{|l|}{ Recompensa } \\
\hline 7. Eu tenho o respeito que mereço dos meus superiores & 0,805 & & & & \\
\hline 8. Eu recebo o respeito que mereço dos meus colegas & 0,879 & & & & \\
\hline 9. Eu recebo apoio adequado em situações difíceis & 0,836 & & & & \\
\hline 10. Eu sou tratado injustamente no trabalho & 0,923 & & & & \\
\hline 11. Minhas perspectivas de promoção no trabalho são poucas & 0,875 & & & & \\
\hline 12. Aconteceu ou está para acontecer uma mudança indesejável na minha situação & & & & & \\
\hline de trabalho & 0,711 & & 0,380 & & \\
\hline 13. Minha garantia no emprego é pequena & 0,842 & & & & \\
\hline $\begin{array}{l}\text { 14. Minha posição atual no trabalho reflete adequadamente meu nível educacional e meu } \\
\text { treinamento }\end{array}$ & 0,366 & & 0,662 & & \\
\hline $\begin{array}{l}\text { 15. Considerando todos os meus esforços e realizações, eu recebo o respeito e o prestígio } \\
\text { que mereço no trabalho }\end{array}$ & 0,658 & & 0,601 & & \\
\hline $\begin{array}{l}\text { 16. Considerando todos os meus esforços e realizações, minhas perspectivas de trabalho } \\
\text { são adequadas }\end{array}$ & 0,492 & & 0810 & & \\
\hline 17. Considerando todos os meus esforços e realizações, meu salário/renda é adequado & 0,415 & & 0,828 & & \\
\hline \multicolumn{6}{|l|}{ Comprometimento excessivo } \\
\hline 18. Eu fico facilmente saturado pelas pressões de tempo no serviço & & 0,610 & & & \\
\hline 19. Eu começo a pensar nos problemas de trabalho logo que acordo de manhã & & 0,808 & & & \\
\hline 20. Quando chego em casa, eu consigo relaxar e desligar-me do trabalho com facilidade & & 0,541 & & & \\
\hline 21. As pessoas próximas de mim dizem que eu me sacrifico demais pelo meu trabalho & & 0,886 & & & \\
\hline 22. O trabalho raramente me deixa. Ele ainda está na minha cabeça quando eu vou para & & & & & \\
\hline $\begin{array}{l}\text { a cama } \\
\text { 23. Se eu adiar alquma coisa que deveria ter feito terei dificuldades para dormir à noite }\end{array}$ & & 0,906 & & & \\
\hline - & & & & & \\
\hline \% da variância explicada & 27,07 & 19,00 & 12,47 & 8,02 & 5,43 \\
\hline
\end{tabular}

eficientes de correlação intraclasse para as dimensões de recompensa e comprometimento excessivo foram superiores a $90 \%$. A consistência interna, medida pelo alfa de Cronbach, foi moderada para a dimensão esforço e ótima para as dimensões recompensa e comprometimento excessivo.

A exploração fatorial (tabela 2) manteve todos os itens iniciais na análise, sendo que cinco fatores explicaram $71,9 \%$ da variância total, como descrito a seguir: Fator 1 , composto pelos itens $7,8,9,10,11,12$, $13,14,15,16$ e 17; fator 2 , composto pelos itens 18, 19, 20, 21, 22 e 23; fator 3, composto pelos itens 14, 16 e 17; fator 4 , composto pelos itens 1, 2, 3, 4 e 6; e fator 5, composto pelo item 5 . A dimensão esforço carregou em três dos cinco fatores, sendo o quarto o fator principal. Os itens componentes da dimensão recompensa carregaram em dois fatores, sendo que a maioria carregou no fator 1 . Os itens 14 , 16 e 17 fazem parte, segundo o construto original, da subescala promoção no emprego/salário, tendo carregado também no terceiro fator. O comprometimento excessivo carregou fortemente todos os seus itens no fator 2 .

\section{DISCUSSÃO}

As publicações existentes sobre o modelo ERI indicam a grande utilidade do mesmo para medir o estresse psicossocial no trabalho. Os dados publicados documentam uma consistência interna satisfatória baseada no alfa de Cronbach, variando entre 0,63 e 0,88 para o componente esforço, de 0,79 a 0,91 para a dimensão recompensa e de 0,74 a 0,78 para o comprometimento excessivo (10-15). No presente estudo, obteve-se um alfa de Cronbach igual a 0,70 para a di- mensão esforço, 0,95 para a dimensão recompensa e 0,86 para a dimensão comprometimento excessivo, todos próximos dos valores mais altos descritos na literatura.

Neste trabalho, no qual o intervalo máximo entre as aplicações da escala foi de 20 dias, a confiabilidade teste-reteste apresentou resultados satisfatórios. Esse curto intervalo parece explicar por que os coeficientes obtidos foram todos superiores a 0,75 , sendo que aqueles referentes às dimensões recompensa e comprometimento excessivo ultrapassaram 0,90, indicando boa confiabilidade da escala. Esses resultados são superiores a outros relatados anteriormente (16).

A estrutura fatorial esteve bem próxima da equivalência, refletindo os componentes do modelo teórico, apesar de alguns itens das dimensões esforço e recompensa terem carregado em mais de 
um fator. A maior carga da questão 5 (esforço físico) em um fator separado se justifica porque a pesquisa foi realizada com um grupo de trabalhadores que praticamente não exerce funções que exijam esforço físico. Os itens de pressão no trabalho, responsabilidades e mais exigências no trabalho, apesar de carregarem no fator 2, podem ser usados como um conjunto na dimensão esforço, porque tiveram carga maior ou bem próxima. A carga dos itens 14 (posição atual no trabalho), 16 (perspectivas no trabalho) e 17 (renda/salário) no fator 3 sugere a possibilidade de análise, em separado, da subescala promoção no emprego/salário, componente da dimensão recompensa, segundo o modelo original (1).

Durante a análise dos resultados da presente pesquisa, foi publicada uma outra versão adaptada para o português brasileiro da escala ERI, que também avaliou suas propriedades psicométri- cas (15). A população participante difere entre os dois estudos, o primeiro tendo sido realizado entre servidores públicos de uma universidade brasileira (15) enquanto que o presente trabalho enfocou bancários de um banco nacional. Existem pequenas diferenças entre as versões, como o uso da palavra "incomodado" em substituição à palavra "estressado" na versão publicada por Chor et al. (15). A apresentação das respostas às perguntas difere um pouco: na versão anteriormente publicada, as respostas são dicotomizadas (concordo/discordo) e em uma delas a frase foi ampliada com as opções "[. . .] e com isso fico: 'nem um pouco estressado, um pouco estressado, estressado e muito estressado'". No presente estudo, as respostas não foram dicotomizadas. Apesar dessas diferenças, os resultados da avaliação são parecidos no que concerne às propriedades psicométricas (validade, consistên- cia interna e construto), sendo que qualquer uma das escalas pode ser usada para estudar situações laborais em português brasileiro.

A principal limitação desta investigação se refere a ter sido realizada com uma única categoria profissional, portanto com menos diversidade de condições que poderiam influenciar potencialmente a adaptação transcultural. Para fazer frente a essa limitação, procurou-se incluir na amostra pessoas com diferentes níveis de escolaridade, faixa etária e funções. De qualquer forma, a realização deste trabalho é importante porque confirma as propriedades psicométricas da escala ERI e indica que ela pode ser usada em estudos ocupacionais no contexto nacional para avaliar o desequilíbrio entre esforço e recompensa, principalmente em populações com características semelhantes àquelas da população aqui analisada.

\section{REFERÊNCIAS}

1. Siegrist J. Adverse health effects of higheffort/low rewards conditions. J Occup Health Psychol. 1996;1(1):27-41.

2. Van Vegchel $\mathrm{N}$, De Jonge J, Bosma $\mathrm{H}$, Schaufeli W. Reviewing the effort-reward imbalance model: drawing up the balance of 45 empirical studies. Soc Sci Med. 2005;60(5): 1117-31.

3. Siegrist J, Starke D, Chandola T, Godin I, Marmot M, Niedhammer I, et al. The measurement of effort-reward imbalance at work: European comparisons. Soc Sci Med. 2004; 58(8):1483-99.

4. Tsutsumi A, Kawakami N. A review of empirical studies on the model of effort-reward imbalance at work: reducing occupational stress by implementing a new theory. Soc Sci Med. 2004;59(11):2335-59.

5. Stansfeld SA, Fuhrer R, Shipley MJ, Marmot MG. Work characteristics predict psychiatric disorder: prospective results from the Whitehall II Study. Occup Environ Med. 1999;56(5): 302-7.

6. Head J, Stansfeld SA, Siegrist J. The psychosocial work environment and alcohol dependence: a prospective study. Occup Environ Med. 2004;61(3):219-24.

7. Siegrist J. Social reciprocity and health: new scientific evidence and policy implications. Psychoneuroendocrinology. 2005;30(10):1033-8.
8. Hasselhorn HM, Tackenberg P, Peter R; NextStudy Group. Effort-reward imbalance among nurses in stable countries and in countries in transition. Int J Occup Environ Health. 2004; 10(4):401-8.

9. Herdeman M, Fox-Rushby J, Badia X. A model of equivalence in the cultural adaptation of HRQoL instruments: the universalist approach. Qual Life Res. 1998;7(4):323-35.

10. Niedhammer I, Siegrist J, Landre MF, Goldberg M, Leclerc A. Psychometric properties of the French version of the EffortReward Imbalance model. Rev Epidemiol Sante Publique. 2000;48(5):419-37.

11. Tsutsumi A, Ishitake T, Peter R, Siegrist J, Matoba T. The Japanese version of the EffortReward Imbalance Questionnaire: a study in dental technicians. Work Stress. 2001;15(1): 86-96.

12. Macías Robles MD, Fernández-López JA, Hernández-Mejía R, Cueto-Espinar A, Rancaño I, Siegrist J. Evaluación del estrés laboral en trabajadores de un hospital público español: estudio de las propriedades psicométricas de la versión española del modelo "Desequilíbrio Esfuerzo-Recompensa". Med Clin (Barc). 2003;120(17):652-7.

13. Li J, Yang W, Cheng Y, Siegrist J, Cho SI. Effort-reward imbalance at work and job dissatisfaction in Chinese healthcare workers: a validation study. Int Arch Occup Environ Health. 2005;78(3):198-204.

14. Eum KD, Li J, Lee HE, Kim SS, Paek D, Siegrist J, et al. Psychometric properties of the Korean version of the effort-reward imbalance questionnaire: a study in a petrochemical company. Int Arch Occup Environ Health. 2007;80(8):653-61.

15. Chor D, Werneck GL, Faerstein E, Alves MGM, Rotenberg L. The Brazilian version of the effort-reward imbalance questionnaire to assess job stress. Cad Saude Publica. 2008; 24(1):219-24

16. Van Vegchel N. Two models at work: a study of interactions and specificity in relation to the Demand-Control Model and the EffortReward Imbalance Model [tese]. Utrecht: Utrecht University; 2005.

Manuscrito recebido em 17 de janeiro de 2009. Aceito em versão revisada em 25 de maio de 2009. 
ABSTRACT Objective. To describe the transcultural adaptation of the effort-reward imbalance scale into Brazilian Portuguese and to examine the resulting construct's validity and reliability.

Transcultural adaptation into Brazilian Portuguese

of the effort-reward imbalance scale: a study with bank workers
Method. Forward and backward translation was used. Internal consistency, testretest reliability, and factor structure were tested in a sample of 100 individuals of both sexes working at a large government-owned Brazilian bank. The sample included different age groups and schooling levels.

Results. The adapted version was named escala de desequilíbrio esforçorecompensa. Cronbach's alpha for the three scale dimensions-effort, reward, and overcommitment-was $0.70,0.95$ and 0.86 , respectively. Intraclass correlation coefficients for these dimensions were $0.82,0.96$, and 0.91 , respectively. Factor analysis kept all the initial questionnaire items and was consistent with the theoretical construct of the model.

Conclusion. The results indicate that the transcultural adaptation into Brazilian Portuguese of the effort-reward imbalance scale was successful and is adequate to assess this situation in work environments.

Key words Stress, psychological; workload; reward; job satisfaction; psychological tests; Brazil. 